\title{
In vivo functional investigations of lactic acid in patients with respiratory chain disorders
}

\author{
G Touati, O Rigal, A Lombès, P Frachon, M Giraud, H Ogier de Baulny
}

Hôpital Robert Debré, Paris, France: Centre for Clinical Investigation G Touati M Giraud H Ogier de Baulny

Biochemistry Laboratory O Rigal

National Institute for Health and Medical Research, Paris, France A Lombès

P Frachon

Correspondence to: Dr Guy Touati, Centre d'Investigation Clinique, Hôpital Robert Debré, 48 Bd Sérurier, 75019 Paris, France.

Accepted 4 September 1996

\begin{abstract}
Objective-To assess the prevalence of in vivo detectable abnormalities of lactate metabolism in mitochondrial disorders.

Design-Retrospective study in a metabolic investigation unit.

Patients-28 patients with a respiratory chain disorder identified from biochemical or genetic analyses, or both, and 133 age matched controls. Controls were children in whom causes of secondary hyperlactataemia and/or disorders, affecting the energy pathways could be excluded.

Methods-Lactate and pyruvate were measured in blood, together with other intermediary metabolism indices, before and one hour after four meals each day. Lactate and creatinine in a 24 hour urine sample collected at the same time were analysed. When basal hyperlactataemia was not evident, an intravenous glucose or pyruvate loading test was performed as a provocative test.

Results-Abnormal lactate metabolism was found in 25 of 28 patients thus demonstrating the potential usefulness of these investigations in the diagnosis of mitochondrial diseases. Moderate lactate accumulation was present in relatively mild disease, associated with a mitochondrial DNA mutation and combined respiratory complexes deficiency. By contrast, high lactate concentrations were observed in very young children, with severe disease, isolated complex deficiency, and no apparent mitochondrial DNA defect.

(Arch Dis Child 1997;76:16-21)
\end{abstract}

Keywords: lactic acidosis; mitochondrial disorders; mitochondrial DNA.

Mitochondrial respiratory chain disorders (RCD), which encompass a large group of diseases, may be difficult to recognise clinically because of their diverse symptoms..$^{1-3}$ Diagnosis is based on histopathological, biochemical, and genetic analyses of mitochondrial dysfunction. These investigations are performed with biopsy samples and may be considered invasive. It is therefore very important to ascertain in vivo mitochondrial dysfunction to help clinical recognition of the disease, as well as to indicate and guide the specialised diagnostic procedures. The main biochemical abnormalities of high lactate concentrations, high lactate/ pyruvate ratios, and high cerebrospinal fluid lactate are considered as the basis of metabolic screening for RCD. ${ }^{4}$ Hyperlactacidaemia may be recorded in the basal state or after a provocative test such as exercise, or intravenous glucose (IVGLT) or pyruvate (IVPLT) loading tests. The prevalence of in vivo detectable abnormalities of lactate metabolism in patients with RCD is difficult to ascertain because in vivo metabolic screening is performed only by some groups and according to different protocols..$^{3-5}$ We have therefore, retrospectively, analysed the results of function tests in 28 patients with RCD and in 133 age matched controls.

\section{Methods}

TECHNIQUES

Blood samples were obtained by venous catetherisation, avoiding hand exercise or a tight tourniquet. Deproteinisation was immediately performed at the bedside, by addition of $0.5 \mathrm{ml}$ whole blood to $1 \mathrm{ml}$ ice cold perchloric acid $(0.9 \mathrm{M})$ and thorough mixing. Samples could then be stored at $-20^{\circ} \mathrm{C}$, for up to 48 hours. Lactate and pyruvate in the body fluids were measured enzymatically. ${ }^{6}$

\section{PROTOCOL OF INVESTIGATIONS}

In the last five years (1990-5), we have examined a total of 243 patients who had a potential defect in their energy metabolism. Informed parental consent was obtained for metabolic investigations in children. All patients were submitted to basal metabolic investigations that started after an overnight fast and lasted for 12 hours in children up to 2 years of age and for 15 hours in older children. Lactate and pyruvate in blood were measured with other intermediary metabolism variables, such as glucose, ketone bodies, free fatty acids, carnitine, insulin, and amino acids. These measurements were performed before and one hour after four daily meals (breakfast, lunch, afternoon snack, and supper). Lactate, creatinine, amino acids, and organic acids in 24 hour urine samples collected at the same time were analysed. Urine was frozen immediately after each urination. We report only the results of lactate, pyruvate, lactate/pyruvate ratio measurements in plasma (L/P), and lactate/ creatinine ratio measurements in urine $(\mathrm{L} / \mathrm{C})$.

When hyperlactacidaemia was not evident, we provocative tests such as IVGLT or IVPLT. These tests were performed only if patients were free of any cardiac insufficiency. Glucose and pyruvate loads were performed after a 12 hour overnight fast. Substrates were injected during a 10 minute period. The glucose load was adjusted to body weight at a dosage of $2 \mathrm{~g}$ glucose/kg body weight in patients whose 
weight was below $25 \mathrm{~kg}$ and $1 \mathrm{~g} / \mathrm{kg}$ body weight in heavier patients. Blood samples were taken before and 15, 30, 60, 90, 120, and 180 minutes after the load. Sodium pyruvate was injected at a dosage of $500 \mathrm{mg} / \mathrm{kg}$ body weight. ${ }^{7}$ Blood samples were taken before and 5, 15, 30, 60 , and 90 minutes after the load. In each test the variables analysed in the blood were glucose, lactate, pyruvate, ketone bodies, insulin, and amino acids. Lactate, creatinine, and organic acids were analysed in urine samples collected six hours after the loads. An IVGLT was mostly chosen as the first provocative test. An IVPLT was mostly used in addition to an ambiguous IVGLT.

\section{RESPIRATORY CHAIN ANALYSIS}

Muscle specimens obtained by open biopsy were from deltoid or quadriceps muscle in all but two patients. Histological and histochemical studies were performed according to described procedures. ${ }^{89}$

Biochemical investigations of the respiratory chain were performed on muscle tissue, cultured skin fibroblasts and, when possible, on others symptomatic organ biopsy specimens (liver, kidney). Enzymatic measurements included polarographic and spectrophotometric analyses. ${ }^{10}{ }^{11}$ The history of each muscle sample allowed exclusion of mishandled or badly preserved specimens.

Southern blot technique allowed analysis of the size and amount of mitochondrial DNA. ${ }^{12}$ In some cases, mitochondrial DNA was also studied by restriction analysis or direct sequencing, or both. ${ }^{12} 13$

\section{CONTROLS}

For ethical reasons, data from functional investigations in normal children could not be established. The diversity of symptoms potentially due to a RCD, however, allowed numerous children with different symptoms, such as mental retardation, hypotonia, growth deceleration, and kidney disorders, to be screened. We could exclude with reasonable certainty the presence of a disorder affecting the energy pathways by analysing intermediary metabolism components measured together with lactate and pyruvate. Children in normal conditions (namely normal nutrition) in whom causes of secondary hyperlactacidaemia (infection, hypoxaemia, and hepatic or circulatory failure) or hyperlactaturia (normal renal function) were excluded were considered as controls. Some 133 patients were retained as controls, when the remaining patients were considered affected with diverse disorders that had metabolic consequences whether or not the disease origin was metabolic. The 133 controls were classified according to age and weight.

PATIENTS WITH RCD

The diagnosis of mitochondrial disorders was based on clinical symptomatology, muscle morphology, and biological investigations, such as enzymatic measurements and genetic analysis. ${ }^{1-3}$
Some 28 patients, older than 1 month of age, had a significant enzymatic deficiency, as found by histochemical or biochemical analysis of the activity of the respiratory chain complexes, or a pathogenic mitochondrial DNA mutation, as found by genetic analysis, or both. Table 1 gives the main patient characteristics. The clinical presentation of some patients correlated with recognised syndromes. Leigh's disease was diagnosed in patient numbers 3, 7, 8, and 9 by the presence of progressive encephalopathy with hyperlactatorrachia and brain basal ganglia alterations as shown by brain imaging. ${ }^{2}$ Myoclonic encephalopathy and ragged red fibres (MERRF) syndrome was diagnosed in patient numbers 17,25 , and 26 by the presence of progressive myoclonic epilepsy with ragged red fibres in the muscle biopsy sample. ${ }^{14}$ These three patients were members of the same family and had different proportions of mitochondrial DNA point mutation associated with the MERRF syndrome. ${ }^{15}$ Myoclonic epilepsy lactic acidosis and stroke-like episodes (MELAS) syndrome was diagnosed in patient number 27 by one stroke-like episode and the presence of progressive mental dysfunction and ragged red fibres in the muscle biopsy specimen. ${ }^{16}$ 'MELAS' syndrome was diagnosed in patient number 23 even though she complained mostly of intolerance to exercise. However, she is the daughter of patient number 27 who has a typical MELAS syndrome and the proportion of the MELAS mutation in a leucocyte DNA sample was similar to that observed in her mother's leucocyte DNA. Kearns-Sayre syndrome was diagnosed in patient numbers 10 , 13,14 , and 16 on onset, before 20 years of age, by ophthalmoplegia with retinitis pigmentosa and hyperproteinorrachia. ${ }^{17}$ However, their first symptomatology was not dominated by their ophthalmoplegia but by intestinal problems in patient number 16 (she has been reported in reference 18), and by deafness and nephropathy in patient numbers 10,13 , and 14. A profound mtDNA depletion quite atypical in Kearns-Sayre syndrome was found in these three latter patients. Encephalomyopathy in patient numbers 2 and 5 had no specific characteristic; it had an early onset and was severe and rapidly progressive. Cardiomyopathy, when present, was always hypertrophic. It was predominant in the clinical presentation of patient numbers 1, 6, and 7. Myopathy in patient numbers $11,12,15,18,19$, and 22 was predominantly expressed as a severe intolerance to exercise. It was later associated with permanent muscle weakness in patient numbers 12, 15, and 22. Ophthalmoplegia in patient numbers $20,21,25$, and 28 was isolated, without any non-muscle symptoms; these patients had ragged red fibres and mitochondrial DNA deletion in their skeletal muscle.

Eleven patients (numbers 1-3, 5-9, 11, 12, and 18) had a profound enzymatic deficiency involving only one of the respiratory chain complexes, the rest of the respiratory chain activities being normal or increased. All these patients had no recognised genetic abnormality. Their mtDNA was of normal size and amount. 
Table 1 Main characteristics of the 28 patients with RCD

\begin{tabular}{|c|c|c|c|c|c|}
\hline Patient No & Age at onset & Age at testing & Main symptoms & Enzyme deficiency & $m t D N A$ \\
\hline 1 & $2 \mathrm{~m}$ & $2 \mathrm{~m}$ & $\mathrm{CM}$ & Complex IV & Normal \\
\hline 2 & $2 \mathrm{~m}$ & $5 \mathrm{~m}$ & Encephalomyopathy & Complex I & Normal \\
\hline 3 & $3 \mathrm{~m}$ & $18 \mathrm{~m}$ & Leigh's syndrome & Complex IV & Normal \\
\hline 4 & $4 \mathrm{~m}$ & $5 \mathrm{y}$ & Nephropathy-CM-deafness & Generalised & Normal \\
\hline 5 & $6 \mathrm{~m}$ & $7 \mathrm{~m}$ & Encephalomyopathy-CM & Complex I & Normal \\
\hline 6 & $8 \mathrm{~m}$ & $5 \mathrm{y}$ & Leigh's syndrome-CM & Complex IV & $\mathrm{ND}^{\star}$ \\
\hline 7 & $9 \mathrm{~m}$ & $4 \mathrm{y}$ & Leigh's syndrome & Complex IV & Normal \\
\hline 8 & $9 \mathrm{~m}$ & $12 \mathrm{~m}$ & Leigh's syndrome & Complex I & Normal \\
\hline 9 & $15 \mathrm{~m}$ & $5 \mathrm{y}$ & Kearns-Sayre syndrome & Complex IV & Normal \\
\hline 10 & $20 \mathrm{~m}$ & $5 \mathrm{y}$ & Kearns-Sayre syndrome & Generalised & Depletion \\
\hline 11 & $2 \mathrm{y}$ & $4 \mathrm{y}$ & Myopathy-deafness & Complex IV & Normal \\
\hline 12 & $3 \mathrm{y}$ & $9 \mathrm{y}$ & Myopathy & Complex IV & Normal \\
\hline 13 & $4 \mathrm{y}$ & $7 \mathrm{y}$ & Kearns-Sayre syndrome & Generalised & Depletion \\
\hline 14 & $5 \mathrm{y}$ & $5 \mathrm{y}$ & Kearns-Sayre syndrome & Generalised & Depletion \\
\hline 15 & $6 \mathrm{y}$ & $20 \mathrm{y}$ & Myopathy-CM & Complex I+IV & Normal \\
\hline 16 & $7 \mathrm{y}$ & $13 \mathrm{y}$ & Kearns-Sayre syndrome & Generalised & Deletion \\
\hline 17 & $8 \mathrm{y}$ & $13 \mathrm{y}$ & MERRF & Complex I+IV & Mutation \\
\hline 18 & $8 \mathrm{y}$ & $18 \mathrm{y}$ & Myopathy & Complex III & Normal \\
\hline 19 & $9 \mathrm{y}$ & $13 \mathrm{y}$ & Myopathy & Generalised & Normal \\
\hline 20 & $12 \mathrm{y}$ & $33 \mathrm{y}$ & Ophthalmoplegia & Complex I+IV & Deletion \\
\hline 21 & $12 \mathrm{y}$ & $13 \mathrm{y}$ & Ophthalmoplegia & Generalised & Deletion \\
\hline 22 & $13 \mathrm{y}$ & $45 \mathrm{y}$ & Myopathy-CM & Complex I+IV & Normal \\
\hline 23 & $14 \mathrm{y}$ & $15 \mathrm{y}$ & MELAS & ND & Mutation \\
\hline 24 & $16 \mathrm{y}$ & $22 \mathrm{y}$ & Ophthalmoplegia & Generalised & Deletion \\
\hline 25 & $18 \mathrm{y}$ & $18 \mathrm{y}$ & MERRF & Complex I+IV & Mutation \\
\hline 26 & $25 \mathrm{y}$ & $41 \mathrm{y}$ & MERRF & Complex I+IV & Mutation \\
\hline 27 & $28 \mathrm{y}$ & $34 \mathrm{y}$ & MELAS & Complex I+IV & Mutation \\
\hline 28 & $30 \mathrm{y}$ & $39 \mathrm{y}$ & Ophthalmoplegia & Complex I+IV & Deletion \\
\hline
\end{tabular}

CM, cardiomyopathy; ND, not determined; $m$, months; y, years.

* Dead affected brother of patient had a normal mtDNA analysis.

In 17 patients there was a combined defect of several complexes of the respiratory chain that coexisted with normal or high values of succinate dehydrogenase and citrate synthase activities (numbers 4, 10, 13-17, and 19-28). Thirteen had a recognised mitochondrial DNA abnormality: depletion in three patients (numbers 10,13 , and 14), deletion in five (numbers $16,20,21,24$, and 28 ) and point mutation in five (numbers 17, 23, and 25-27).

\section{Results}

CONTROLS

Among the 243 patients, older than 1 month of age, who were investigated in the last five years, 133 were considered retrospectively as controls. They were classified into three groups according to age and weight: group 1 comprised 45 infants with a median age of 9.6 (range 2-23) months and median weight of 8.15 (range 3.4-15.0) kg; group 2 comprised 65 children with a median age of 4.7 (range 2.0-9.7) years and median weight of 16.4 (range 8.5-46.2) kg; and group 3 comprised 23 patients with a median age of 14.8 (range 10-41) years and median weight of 51.5 (range $22-105) \mathrm{kg}$. Adults and children older than 10 years of age were pooled because their blood lactate concentrations, blood $\mathrm{L} / \mathrm{P}$ ratios, and urinary $\mathrm{L} / \mathrm{C}$ ratios were in the same range (data not shown).

Tables $2-4$ give the results of investigations in the basal state, and after an IVGLT and an IVPLT, respectively. Data from the IVPLT were pooled as this test was performed in only 14 controls, whose age ranged from 13 months to 15 years: three patients were younger than 2 years of age, nine were between 2 and 10 years, and two were older than 10 years.

In basal investigations (table 2), controls in group 1 seemed to have a significantly higher lactate concentration than older children (groups 2 and 3) ( $\mathrm{p}<0.01$, unpaired Student's $t$ test) in the fasted as well as in the fed state. This difference was not significant between controls in groups 2 and 3. The dispersion of the values was also significantly higher in the controls in group 1 as compared with that in older children $(\mathrm{p}<0.001$, analysis of variance).

However, induced lactate accumulation during the IVGLT was not significantly different in the controls in group 1 as compared with that in group 2 ( $\mathrm{p}>0.05$ Student's $t$ test). When the results were compared with controls in group 3 , the lactate concentrations were significantly higher in infants at 90 and 120 minutes $(\mathrm{p}<0.05)$ but not at other times.

\section{PATIENTS WITH RCD}

Twenty patients (numbers 1-9, 12-19, 22, 25, and 26) had a permanent hyperlactataemia (their lactate was above the 90th centile of normal values in more than half of their analyses). Their lactate concentrations were relatively high (above $2.5 \mathrm{mmol} / 1$ in $87 / 156$ samples and above $4 \mathrm{mmol} / 1$ in 38/156 samples). All these patients had high $\mathrm{L} / \mathrm{P}$ ratios and all but six (numbers 6, 14, 15, 22, 25, and 26) also had high L/C ratios. Despite this significant basal hyperlactataemia nine of these patients had provocative tests: an IVGLT in nine and an IVPLT in three of them. These IVGLT and IVPLT were always abnormal, with significant hyperlactataemia. Hyperlactaturia was also present in all patints who underwent an IVPLT and in seven of nine who had an IVGLT.

Many of the 20 patients had a very young age of onset, before 10 years of age in 17. Six have died and 12 are severely handicapped by their disease. Ten of these patients had a profound biochemical defect in one of the respiratory chain complexes, without a concomitant mtDNA abnormality. An inherited autosomal recessive disorder was supected in nine of these 10 patients (consanguineous parents, or affected siblings born to healthy parents). 

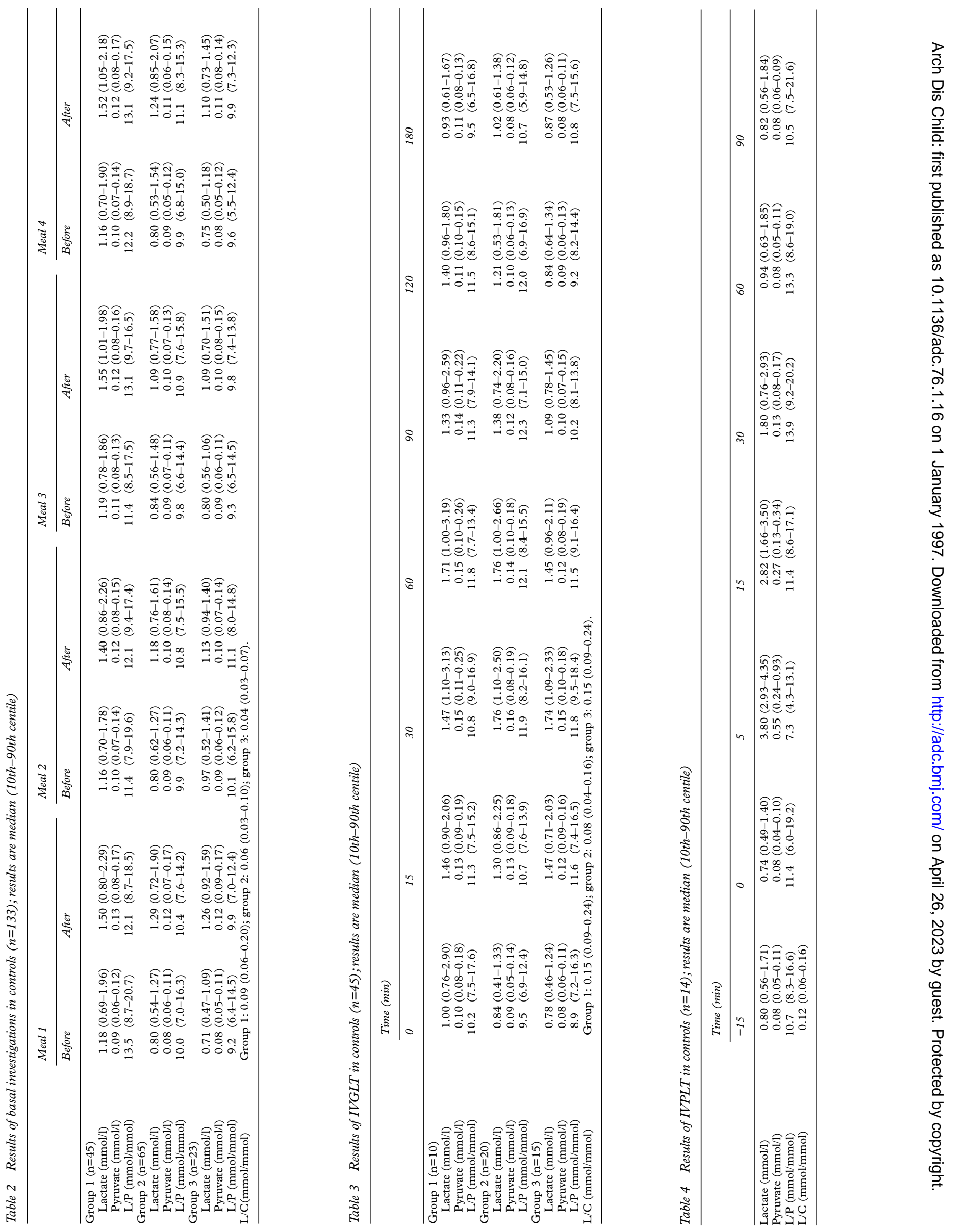
The remaining eight patients (numbers 10, $11,20,21,23,24,27$, and 28) had no permanent abnormality in their basal investigations. Four (numbers 10, 11, 20, and 24) had intermittent (less than $50 \%$ of the measurements above the 90th centile of normal values) and moderate (above $2.5 \mathrm{mmol} / 1$ lactate in two of 31 samples and above $4 \mathrm{mmol} / 1$ in none of 31 samples) hyperlactataemia. These four patients were submitted to an IVGLT. Induced lactataemia was normal in patient number 24 who was not submitted to an IVPLT, while it was abnormal in the three remaining patients (10, 11, and 20). Patient numbers 10 and 11 also showed significantly induced hyperlactaturia. An IVPLT performed in two patients (numbers 10 and 20) induced abnormal hyperlactataemia and hyperlactaturia. One patient (number 27) had normal basal concentrations of lactate contrasting with $\mathrm{L} / \mathrm{P}$ and $\mathrm{L} / \mathrm{C}$ ratios slightly above the 90th centile of controls values. This patient did not undergo an IVGLT but an IVPLT showed significant hyperlactataemia, and high $\mathrm{L} / \mathrm{P}$ and $\mathrm{L} / \mathrm{C}$ ratios. The three remaining patients (numbers 21, 23, and 28) had normal lactate, and normal $\mathrm{L} / \mathrm{P}$ and normal L/C ratios. An IVGLT was performed in these three patients and an IVPLT in one. The results of these tests were normal.

The eight patients without permanent abnormality in their basal metabolic investigations had a relatively late age of onset (mean 174 months (14.5 years), range 20 months-30 years). None has died and six are adults with a normal social life. Seven of these eight patients has an identified mitochondrial DNA mutation associated with combined defects in respiratory chain activities (table 1 ).

\section{Discussion}

This retrospective study was possible only by adopting a strict methodology in the testing procedures, to obtain reliable control values. Our choice of controls is open to criticism. Normal children will obviously not undergo metabolic screening. All the control children presented with symptoms potentially attributable to a RCD or other intermediary metabolism disorder. These conditions were excluded. The diversity of symptoms allowed numerous children to be screened and thus some of the obvious sampling bias to be avoided. The relative narrowness of our control values may be considered as a retrospective indicator of the appropriate choice of controls.

Lactate concentrations in the controls in group 1, however, were higher and more dispersed than in the patients of groups 2 and 3. This larger range may be due to the inclusion of some false controls as it is clearly more difficult to exclude all causes of secondary hyperlactataemia, especially as a result of difficulties in blood withdrawal, in very young children. It could also reflect the dispersion of physiological metabolic maturation in very young children. Whatever its cause, this dispersion has already been reported ${ }^{6}$ and further emphasises the necessity of age matched control groups, at least for infants. Observed differences between groups 2 and 3 are less obvious and separation of these two groups may be futile. This conclusion, however, has to wait for the inclusion of a greater number of controls.

Lactate concentrations and $\mathrm{L} / \mathrm{P}$ and $\mathrm{L} / \mathrm{C}$ ratios were analysed in basal metabolic investigations. These variables seemed to be useful in the assessment of patients. Lactate was the most sensitive indicator. However, the $\mathrm{L} / \mathrm{C}$ ratio was very beneficial in some instances. Three patients (numbers 10,13, and 14) had a renal disorder with a low level of tubular lactate reabsorption that could lead to normal lactate and high $\mathrm{L} / \mathrm{C}$ ratios. Two patients (numbers 20 and 27), with no apparent kidney disorder, had $\mathrm{L} / \mathrm{C}$ ratios above the 90th centile when their lactate was normal. Furthermore, measurement of $\mathrm{L} / \mathrm{C}$ ratios is not prone to false positive results due to difficulties in blood sampling and deproteinisation. The usefulness of $\mathrm{L} / \mathrm{P}$ ratios is obvious in the differential diagnosis of hyperlactataemia due to pyruvate dehydrogenase complex deficiency. However, pyruvate concentrations are highly sensitive to blood sample preparation, giving rise to artificially low pyruvate concentrations and therefore to false high $\mathrm{L} / \mathrm{P}$ ratios. The $\mathrm{L} / \mathrm{P}$ ratio was a sensitive variable in our series of patients with RCD. It was always abnormal when blood lactate was above $2.5 \mathrm{mmol} / 1$ and two patients (numbers 24 and 27) showed high L/P ratios concomitantly with normal lactataemia.

Metabolic abnormalities were present in the basal state in 25 patients. In 14 patients, however, they were considered to be disputable and therefore a provocative test was performed. This test showed significant abnormalities in all except one patient (number 24). Our recognition of the significance of these abnormalities in the basal state has evolved with the progressive accumulation of data. Some early patients who had a provocative test would not be given it now.

In this study, there was a high prevalence $(25 / 28)$ of in vivo detectable abnormalities of lactate metabolism in patients with authentified RCD. Analysis of lactate metabolism, among other characteristics, was recently reported in a large series of patients with $\mathrm{RCD},{ }^{3}$ using a unique determination of lactatein the fasted state. Hyperlactataemia was found in 21 of 47 patients, in particular in 15 of the 20 children included in the study. The authors' concluded that this variable has little value as a screening test for RCD. Using systematic evaluation of blood lactate, within a day, in both the fasted and fed state, we obtained a significantly higher prevalence of hyperlactataemia that considerably improves the potential usefulness of lactate analysis in the screening for RCD.

Some patients with RCD, however, will show normal lactate metabolism in the basal state as well as in intravenous loading tests. In our series four patients $(21,23,24$, and 28$)$ did not show any significant defect of their lactate metabolism after such a test. These four patients had a mitochondrial DNA mutation and a mild clinical phenotype. Interestingly, the paper by Jackson et al also stated that lactate 
measurement was of little value in patients presenting with chronic external ophthalmoplegia, ${ }^{3}$ which is frequently associated with a mitochondrial DNA mutation. Mitochondrial dysfunction in these patients is possibly mild enough not to give any measurable disorder of a plasma variable. By contrast, if the load on lactate metabolism induced by our provocative tests was clearly insufficient, another type of provocative test (such as an exercise test on an ergometric bicycle) might induce some significant abnormal response. Such exercise tests were not performed and therefore their provocative efficiency relative to that of intravenous loading tests cannot be established.

Hyperlactataemia was not only useful for the clinical recognition of a RCD, but it also had prognostic value. In particular, none of our most severely affected patients had normal metabolic results in the basal state and all patients with normal metabolic findings had the mildest clinical phenotype (symptoms mild and involving only muscle). Furthermore, the reported metabolic investigations were performed during the initial diagnostic steps. Some patients have been followed up for years during which time we have seen a progressive increase in lactate concentrations in the basal state together with a continuing deterioration in clinical status.

One result of the study has been to strengthen our belief that some patients (not described here) are affected with a 'yet to be demonstrated RCD'. These patients did not present with the histochemical, biochemical, or genetic diagnostic criteria of a RCD but had hyperlactataemia associated with abnormal redox ratios. There are numerous reasons as to why a RCD cannot be assessed. For example, morphological and biochemical analyses are mostly performed using muscle samples and the muscle may be spared in some patients with the disease; only relatively profound deficiencies of the respiratory chain are found with the usual biochemical analyses and not all the complexes are systematically analysed; and mitochondrial DNA is not sequenced in every patient, especially when there is no maternal inheritance of the disease, therefore point mutations may be easily ignored.
We are indebted to Dr Norma B Romero, Marie A Cheval, and to the nursing staff of the Centre d'Investigation Clinique for their contribution in the morphological analysis and their nursing care. This work was supported by grants from the Association Française contre les Myopathies and by the Institut National de la Sante et de la Recherche Medicale.

1 DiMauro S, Moraes CT. Mitochondrial encephalomyopathies. Arch Neurol 1993;50:1197-208.

2 DeVivo DC, DiMauro S. Mitochondrial diseases. In: Swayman KF, ed. Pediatric neurology. Principles and practice. St Louis, Missouri: Mosby-Year Book, 1994:1335-56.

3 Jackson MJ, Schaefer JA, Johnson MA, Morris AAM, Turnbull DM, Bindoff MA. Presentation and clinical investigation of mitochondrial respiratory chain disease. A study of 51 patients. Brain 1995;118:339-57.

4 Trijbels JMF, Sengers RCA, Ruitenbeek W, Fischer JC, Bakkeren JAJM, Janssen AJM. Disorders of the mitochondrial respiratory chain: clinical manifestations and diagnostic approach. Eur f Pediatr 1988;148:92-7.

5 Tulinius MH, Holme E, Kristiansson B, Larsson N, Oldfors A. Mitochondrial encephalomyopathies in childhood. I. Biochemical and morphologic investigations. 7 Pediatr 1991;119:242-50.

6 Vassault A, Bonnefont JP, Specola N, Saudubray JM. Lactate, pyruvate and ketone bodies. In: Homme FA, ed. Techniques in diagnostic human biochemical genetics. A laboratory manual. New York: Wiley-Liss, 1991:285-308.

7 Dijkstra U, Gabreëls F, Joosten E, et al. Friedreich's ataxia: intravenous pyruvate load to demonstrate a defect in pyruvate metabolism. Neurology 1984;34:1493-7.

8 Dubowitz V, Brooke MH. Muscle biopsy: a modern approach. Philadelphia: WB Saunders, 1973.

9 Seligman AM, Karnovsky MJ, Wasserkrug HL, Hanker JS. Non-droplet ultrastructural demonstration of cytochrome oxidase activity with a polymerising osmiophilic reagent, diaminobenzidine (DAB). f Cell Biol 1968;38:1-14.

10 Letellier T, Malgat M, Coquet M, Moretto B, ParrotRoulaud F, Mazat JP. Mitochondrial myopathy studies on permeabilized muscle fibers. Pediatr Res 1992;32:17-22.

11 Rustin P, Chretien D, Bourgeron T, et al. Biochemical and molecular investigations in respiratory chain deficiencies. Clin Chim Acta 1994;228:35-51.

12 Zeviani M, Moraes CT, DiMauro S, et al. Deletions of mitochondrial DNA in Kearns-Sayre syndrome. Neurology 1988;38:1339-46.

13 Gyllensten UB, Erlich HA. Generation of single-stranded DNA by the polymerase chain reaction and its application to direct sequencing of the HLA-DQA locus. Proc Natl Acad Sci U S A 1988;85:7652-6.

14 Fukuhara N, Tokiguchi S, Shirakawa K, Tsubaki $\mathrm{T}$. Myoclonus epilepsy associated with ragged-red fibres (mitochondrial abnormalities): disease entity or a syndrome? F Neurol Sci 1980;47:117-33.

15 Shoffner JM, Lott MT, Lezza AMS, Seibel P, Ballinger SW, Wallace DC. Myoclonic epilepsy and ragged-red fiber disease (MERRF) is associated with a mitochondrial DNA tRNAlys mutation. Cell 1990;61:931-7.

16 Pavlakis SG, Phillips PC, DiMauro S, DeVivo DC, Rowland LP. Mitochondrial myopathy, encephalopathy, lactic acidosis, and stroke like episodes: a distinctive clinical syndrome. Ann Neurol 1984;16:481-8.

17 Rowland LP. Progressive external ophthalmoplegia. In: Vinken PJ, Bruyn GW, DeJong JMBV, eds. Handbook of clinical neurology. Part II: system disorders and atrophies. New York: American Elsevier Publishing, 1975:177-202.

18 Cormier-Daire V, Bonnefont JP, Rustin P, et al. Mitochondrial DNA rearrangements with onset as chronic diarrhea with villous atrophy. $\mathcal{F}$ Pediatr 1994;124:63-70. 